\title{
Perceptions of stereotypes applied to women who publicly communicate their STEM work
}

\author{
Merryn McKinnon (1D ${ }^{1,3 凶} \&$ Christine $\mathrm{O}^{\prime}$ Connell ${ }^{2,3}$
}

Gender biases and stereotypes are prevalent in science, technology, engineering and mathematics (STEM) fields, which can create obstacles for the attraction, retention and progression of girls and women to STEM studies and careers. There are many initiatives which are used to attempt to address these biases and stereotypes, including the use of visible role models. This study explores the perceptions of the stereotypes applied to female STEM professionals who publicly speak about their work in both academic and non-academic settings. Using workshops with over 300 participants, predominantly female STEM professionals, from over 25 different cultural backgrounds, the results showed women who publicly communicate their work are likely to be stereotyped as 'bitchy', 'bossy', and 'emotional'often by their own gender. These findings suggest that women may be in a more vulnerable position when communicating publicly about their work, which could have implications for them participating fully in their careers. It may also have implications for programs which use role models to address prevailing STEM stereotypes. Systematic cultural and institutional change is needed in STEM fields to address the underlying bias and negative stereotypes facing women. However, it should be ensured that the intended solutions to facilitate this change are not compounding the problem.

\footnotetext{
${ }^{1}$ Centre for the Public Awareness of Science, Australian National University, Canberra, ACT 2601, Australia. ${ }^{2}$ School of Journalism and the Alan Alda Center for Communicating Science, Stony Brook University, Stony Brook, NY 11794, USA. ${ }^{3}$ These authors contributed equally: Merryn McKinnon, Christine

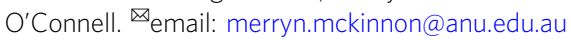




\section{Introduction}

ender gaps and bias are still prevalent in science, technology, engineering and mathematics (STEM) fields. Gender-based stereotypes present obstacles for women in STEM and continue to play a role in ongoing discrimination and under-representation of women in STEM professions (Carli et al., 2016). Experiences with gender bias and stereotypes in STEM are compounded throughout female scientist's careers and have been shown to emerge as early as kindergarten (Bian et al., 2017; Cimpian et al., 2016). One dominant stereotype is that boys are better at maths and science than girls, which studies show is not true (O’Dea et al., 2018). In addition, stereotypical traits of scientists such as objectivity and rationality are generally consistent with male gender-normative traits (Settles et al., 2016). Comparatively, women are seen as highly communal (i.e., kinder, warmer, empathetic) and less agentic (i.e., analytical, independant, and competitive) and therefore less likely to have the qualities and personality characteristics needed to be successful scientists (Carli et al., 2016). Gender based stereotypes like this not only influence the career choices for women in STEM, but also the retention of women in STEM fields as they must overcome deeply embedded discrimination and bias (Ellemers, 2018; Carli et al., 2016).

These gendered STEM stereotypes are inherently influenced by the dominant social norms that position STEM as being maleoriented (Garriott et al., 2017), including in media coverage of science and technology (Women's Leadership Institute Australia, 2019). Public perceptions of science are shaped by exposure to science and role models, and the cultural contexts and beliefs of the individuals who are exposed (Noy and O'Brien, 2019). Role models can be used to address negative stereotypical perceptions of women in STEM fields, as well as both attract and retain girls and women in STEM studies and careers (Drury et al., 2011; Shin et al., 2016). However what are the implications of being a visible woman in STEM? This study aims to provide insight into the perceptions of the stereotypes which are applied to women who speak publicly about their research and/or work. The implications of these stereotypes for women in STEM, particularly those who may be considered role models, are then discussed.

\section{Literature review}

Gender stereotypes are shared by all and tend to oversimplify reality, creating judgments of people based on perceived-rather than actual-ability (Ellemers, 2018). Women are stereotypically defined as having communal traits such as being warm and nurturing, whereas men are stereotypically allocated agentic traits such as competence and assertiveness (Fiske et al., 2002) with the latter considered much more consistent with competitive STEM fields (Settles et al., 2016). If a group is negatively stereotyped (e.g., seen as somehow lesser), membership of this group can influence psychological health (Roberts et al., 2008), performance (Steele, 1997) and one's sense of identity and belonging (Diekman et al., 2019). This is especially the case if the role models have high levels of stigma consciousness; an expectation of judgment because of a specific group membership, irrespective of behavior or performance (Pinel, 1999). Stigma consciousness may be an important indicator of vulnerability of women to the negative influence of stereotype threat in real world contexts (Cadaret et al., 2017).

Awareness of being negatively perceived or 'other' can influence identity, either identity as a particular gender or as someone who does science (Carlone and Johnson, 2007). Earlier studies have also found that members of marginalized groups may further penalize those who display these marginalized identity traits (Opie and Phillips, 2015). Therefore, members of marginalized groups may be reluctant to display the traits which identify them as a member of that group, negating attempts to increase workplace diversity and inclusion (Opie and Phillips, 2015). For example, studies have found that women in academia are evaluated more on personality then ability, compared to male colleagues, and are expected to be more nurturing and empathetic (Heilman and Okimoto, 2007; Mitchell and Martin, 2018). Not only do these stereotypes have implications for women's careers if they do not conform to being 'warm', but as a marginalized group, they may penalize other women who display this stereotype (Opie and Phillips, 2015). In addition, accomplishments that challenge the stereotype (e.g., women being good at science) are often discounted or attributed to outside help (Ellemers, 2018).

Stereotypes lead people to treat men and women differently, hold them to different standards, and perpetuate gender bias in society, including in STEM (Ellemers, 2018). These perceptions and evaluations are underpinned by both explicit and implicit biases, with the latter more automatic and less conscious and controllable than the former (Charlesworth and Banaji, 2019). Previous studies have shown that even women working within science hold an implicit stereotype of science being a masculine area and women 'belonging' in the arts (Smyth and Nosek, 2015). Implicit biases which reinforce gender stereotypes have been found to become evident from a young age and appear across genders, cultures and time (Charlesworth and Banaji, 2019).

Gender stereotypes negatively influence a women's perceived potential, and also how they are evaluated (Ellemers, 2018) including in job materials like reference letters and resumes (Milkman et al., 2015; Moss-Racusin et al., 2012). In teaching evaluations specifically, women experienced a negative bias in ratings and were evaluated on different criteria than men, including appearance and personality (Mitchell and Martin, 2018). Articles are cited less when key authors are female, and research has shown an underrepresentation of female authorships in prestigious journals (Bendels et al., 2018). Despite this body of research, there are some men-and probably some women-in STEM faculties who are reluctant to accept research and evidence of gender bias in STEM (Handley et al., 2015). However, gender biases can all have serious and lasting impacts on the person targeted and the cumulative effects can be extremely damaging to both their career and self (Knobloch-Westerwick et al., 2013; National Academies of Sciences, 2018). Women who publicly communicate their work may be especially vulnerable to these different types of harassment. Gender-based stereotypes can amplify harrassment and discrimination in STEM, especially when those stereotypes about women do not overlap with those about perceptions of what it means to be a scientist (Carli et al., 2016).

Gender bias and sexism are pervasive and well documented in STEM fields, disproportionately disrupting and impacting women (National Academies of Sciences, 2018; Rosenthal et al., 2016) especially women of color (Clancy et al., 2017) and LGBTQ+ and non-binary individuals (Konik and Cortina, 2008). The most common type of harassment in STEM fields is gender harassment (National Academies of Sciences, 2018), which can involve disrespectful actions, put downs and negative comments that convey the false narrative that women are lesser than their male counterparts (National Academies of Sciences, 2018; Weitz, 2018). In disciplines like engineering, they can suffer from (in)visibility where they are highly visible as a woman but their abilities as engineers are contested (Faulkner, 2009). Then there are Queen Bees, even in non-STEM fields; women who have risen to leadership positions in male dominated organizations and then distance themselves from junior women, agree with negative 
Table 1 Examples of reinterpreted (flipped) stereotypes.

\section{Stereotype (negative or positive) Reinterpretation}

Bitchy (-)

Soft-spoken (-)

Straightforward, commanding. Example: In a situation where a researcher must stop an action (e.g., if someone is acting inappropriately or dangerously in the lab), one might choose to be clear and project an assertive and commanding voice and body language.

Motherly (-)

Allows space for other voices to be heard. Example: In a situation where someone wants to encourage those around them to speak their opinions and contribute to a discussion, sometimes we have to lower our own voice.

Provides a healthy and supportive environment where students thrive

Too pretty $(-)$

Put together and professional; comfortable and confident

Distracted by things that are not valued in tenure decisions. Example: In a situation where an assistant professor is up for tenure, but spending a large portion of their time on service oriented activities, which takes away from the amount of time they have left to spend on getting grants or writing peer reviewed publications (things that are more valued in the tenure process); this might negatively impact their getting tenure.

stereotypes and effectively reinforce gender inequality (Derks et al., 2016).

The negative effects of gender-based stereotypes extend to public communication activities of women in STEM. Media worldwide have traditionally under-represented females in STEM, and where they were represented the focus tended to be either on their appearance or their feminine roles as wives and mothers (Chimba and Kitzinger, 2010). Even in Finland, which has a strong commitment to gender equality, the media predominantly interview male scientists as experts (Niemi and Pitkänen, 2017). Women experience negative stereotypes and bias when talking or writing about their work, including in teaching and presentations (Mitchell and Martin, 2018), research articles (Budden et al., 2008), and in YouTube and social media (Amarasekara and Grant, 2019; Veletsianos, 2012). But what perceptions do women in STEM have of the stereotypes which might be applied to those who speak publicly about their work?

In this study we used workshops to examine participant perceptions of which stereotypes are applied to women who speak publicly about their research or work and explore whether these stereotypes are perceived as positive or negative. Understanding the perceptions of stereotypes applied to those who publicly communicate their science allows a more nuanced understanding of the potential impact being visible or being a role model may have. We also outline how this understanding may enable the development of better mechanisms to support women in STEM.

\section{Methods}

Participants were recruited via email, website, newsletter notices, conference sessions and direct requests for workshops by the researchers. Recruitment was initially within the researchers' home institutions but then expanded to their networks and via word of mouth. Stereotype data was collected from September 2017 until April 2018 within workshops conducted in various locations in Australia, New Zealand, USA and Japan. These workshops were conducted during conferences, women in STEM leadership/professional development programs and in sessions specifically scheduled for this research. Ethics clearance for this research was obtained from both researchers' home institutions and all participants provided informed consent.

The workshops. The workshops ranged in size from four to 80 participants at a time. The larger workshops were broken into smaller groups (up to 10 people) for the data collection exercise, resulting in 49 distinct groups used for data collection across all 17 workshops. Each workshop followed a similar structure. After a brief introduction, large sheets of paper and colored pens were distributed to tables around the room. Working in the smaller focus groups, participants were asked to write down "all of the gender based stereotypes that are applied to women who communicate about their science [research/work]". After $15 \mathrm{~min}$, participants were then instructed to mark stereotypes as positive or negative. Participants may have had some disagreement about whether particular stereotypes were positive or negative and were instructed that this was okay and to mark these stereotypes as "both". This positive/negative perception section elicited more discussion and storytelling within each group, so was typically allocated about $25 \mathrm{~min}$. Timing for both parts could be lengthened or shortened depending upon the total time available and the nature of the engagement in the room.

Once stereotypes had been listed as positive, negative or both, groups came back together and a whole room discussion was held. One group would start to read their list of negative stereotypes. As each stereotype was read, it would be described (including use of participant derived examples if necessary for clarity) and then the facilitator would lead a short discussion on how this stereotype could be 'flipped'-made positive or negative depending on the situation, audience, and goal of the communicator. Examples of stereotype 'flips' are provided in Table 1. Each group marked off the corresponding negative stereotype on their own list so as one group finished their sheet of paper, others may have had only a few additional stereotypes. These were also discussed until all stereotypes generated by participants had been covered (time permitting). At the end of the workshop the sheets of paper were collected and retained for analysis.

Data analysis. Both researchers coded the collected sheets of paper using a cross sectional 'code and retrieve' method (Mason, 2002). Each stereotype listed was entered into a spreadsheet and then iteratively grouped into similar themes of analytical categories through a process of inductive category development (Mayring, 2000), which is appropriate given the limited existing knowledge (Elo and Kyngäs, 2008) about perceptions of stereotypes for women in STEM publicly communicating their work. As the data consisted of single words or short phrases, each individual word was read and used to derive the code categories (Miles and Huberman, 1994). The number of times a stereotype theme appeared on each page was also counted. For example 'judged by appearance', 'too much makeup', 'too young' would all be categorized within the theme 'judged' and counted as the judged stereotype appearing three times on that sheet.

The researchers each coded the sheets each after the first workshop and refined the categories together to ensure consistent categorization. The coding process was repeated at different times during the data collection, when approximately half the data was entered and again once all data was entered. The repetition allowed for a systematic overview of all the data collected and the development of thematic categories which best represented the data as a whole. Throughout the process, exemplars of each category were identified from the data (Hsieh and Shannon, 
2005) and relationships between them clarified which refined the total number of categories. Any stereotypes which were interpreted differently by the researchers were discussed, resolved, and subsequently consistently coded using the validated definition.

As the purpose of this study is to explore perceptions of stereotypes, the categories have been left intentionally broad to reflect the diversity and complexity of the participants' perspectives (Hsieh and Shannon, 2005). Analysis was limited to manifest, rather than latent, content meaning as the researchers could only be certain of the visible, surface content of text (Cho and Lee, 2014). The latent or underlying meaning (Graneheim and Lundman, 2004) could not have been interpreted with reasonable accuracy as little was known about the participants as individuals or the context of the conversations from which the single words or short phrases were derived. As this study is intended to be descriptive, staying to the "...surface of words..." (Sandelowski, 2000 , p. 336) was deemed the most appropriate analytical approach. Validity of the data collected is supported through the extended time spent debriefing and discussing the words and phrases elicited from participants during the workshops, ensuring that their intent and meaning was understood and accurately interpreted (Manning, 1997). To the authors' knowledge this is one of, if not the only, attempt to capture perceptions of stereotypes applied to the public communication activities of women in STEM. Therefore the intent of this analysis is to present a preliminary development of the concept or model (Lindkvist, 1981) rather than a deeper analysis of lived experience or development of theory which grounded theory or alternative form of qualitative analysis would offer (Hsieh and Shannon, 2005).

\section{Results}

Stereotype categories. About 315 people, the vast majority women, participated in the workshops with representatives from around 25 different cultural backgrounds identified. In total, data was collected from 49 different groups within 17 workshops. Participants tended to range from early career through to senior levels with the majority from within academia. Specific demographic data was not collected-identification of different demographic variables was made through either recruitment processes, workshop location or self-identification by participants during discussions.

Participant responses yielded 1273 words or phrases which were then coded into a total of 17 categories. While these categories could potentially be refined further, some of the nuance would be lost. Each category, and indicative words used in the generation of that category, are shown in Table 2. Each category name is deliberately neutral rather than directional, even if seemingly obviously negative, as participants were asked to describe whether these are positive or negative based on their perception, as described later. Emotional and empathetic were kept separate as the emotional stereotype category typically reflected hysterical or irrational responses, whereas the empathetic stereotype category dealt more with interpersonal skills and maternal tendencies. Credibility, worth and undeserving also appear closely related but the differences are quite specific. Credibility focuses on whether women are seen as competent and experienced or as somehow lesser than their male counterparts. Worth specifically refers to whether women are perceived as being worthy of support, professional development or any other form of investment from employers. Undeserving is tied to credibility but was kept within its own category due to the comparatively common responses related to women being lucky to have achieved what they have, and the implication that sexual or other favors must be related to the woman's success.
Perceptions of stereotypes. All individual words and phrases were counted, coded and recorded as they were indicated by the attendees to be considered positive, negative or both. The most commonly occurring category was 'Bitchy', with stereotypes from that category appearing 167 times across all groups; just over 13\% of responses (Table 2). 'Credibility' was the next most commonly appearing category with 143 mentions (11.2\%) and 'Appearance' in third with 130 mentions (10.2\%, Table 2). Participants shared stories and examples during this exercise of how they changed their behavior based on the stereotype. For example, in naming a stereotype in the category of 'Appearance' one participant admitted to not wearing skirts at conferences or speaking engagements anymore after hearing a male scientist make a comment about a female speaker's legs while she was on stage. Another said she purposely did not wear a lot of make-up, put her hair up and wore her glasses when she taught or gave presentations, so she would not look 'pretty' and would therefore be taken more seriously.

Unsurprisingly, the identified stereotypes were predominantly considered negative, especially those in the credibility, judged on appearance, annoying, confidence, worth, and undeserving categories (Fig. 1). Categories that were considered to be more positive included empathetic, nice, efficient, collaborative and superwoman, however participants indicated that these could also be considered negative, particularly the empathetic and nice categories (Fig. 1). The negative nature of the stereotype is reflected in some of the words and phrases consistently being raised in the focus groups. A word cloud generated using all words and phrases collected and used in the coding process clearly shows 'bossy', 'bitchy', and 'emotional' as the most commonly occurring (Fig. 2).

An interesting word that was commonly elicited in all workshops was 'too'. Usually this was in the context of 'too many', 'too much', or 'too little' and occasionally replaced or used in conjunction with 'overly'. Typically, these were used in relation to women's behaviors, for example asking questions (too many), being emotional, assertive or ambitious (overly/too), or their general appearance (too pretty) including make up (too much/ little) and age (too old/young). The other words that surfaced attached to a stereotype were 'more' or 'less' (e.g., more motherly than, less serious than); 'enough' or 'lack of (e.g., not smart enough, lack of drive) and 'not as' or 'overly' (e.g., not as friendly as or overly friendly).

Confronting personal bias. After identifying the dominant stereotypes, each group was then led through an exercise to 'flip' the stereotype (described further in "Methods" section). The 'flipping' exercise created discussion amongst the participants of how they may be able to respond rather than react to the stereotype and deflate the negative connotation of the stereotype by making a measured communication choice. For example, the majority of the group had witnessed a situation when a woman was referred to as 'bitchy', or some other derogatory word, when she spoke her mind or stood up to someone in an assertive way; they admitted to this shaping their behavior in the future. Participants discussed the implications of when individuals make communication decisions based on a perceived stereotype versus the situation at hand.

When looking at a stereotype as positive or negative, and then flipping it, participants started to look at how they labeled not just themselves but others in various situations; they were forced to confront their own bias. During the 'flipping' exercise several participants, in multiple workshops, admitted to feeling annoyed by other women who demonstrated a stereotype. For example, in situations where a woman was soft-spoken or shy, some shared 
Table 2 Stereotype categories, with indicative samples, identified through the coding of focus group responses.

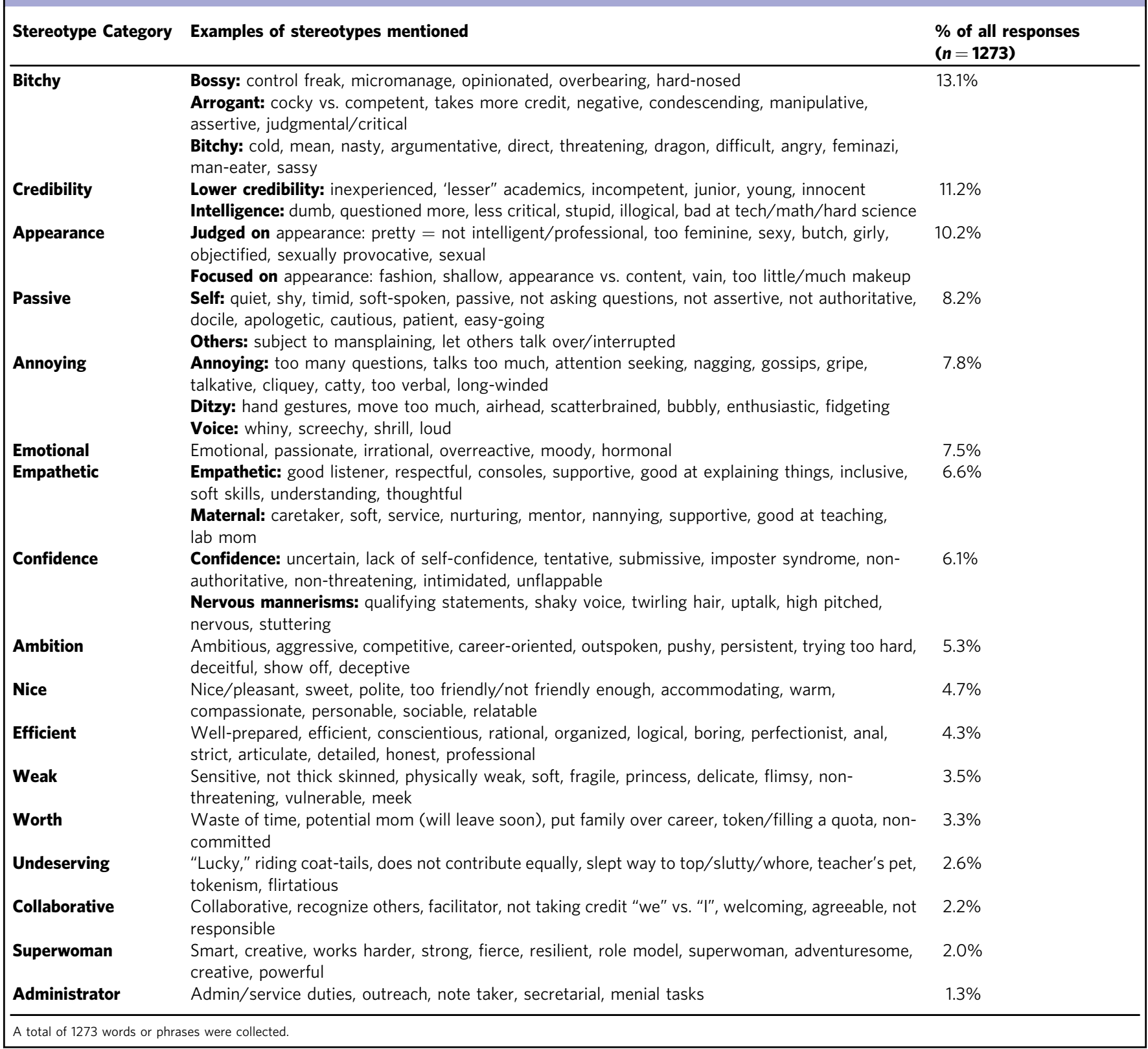

Perception of Stereotype (positive, negative, or both)

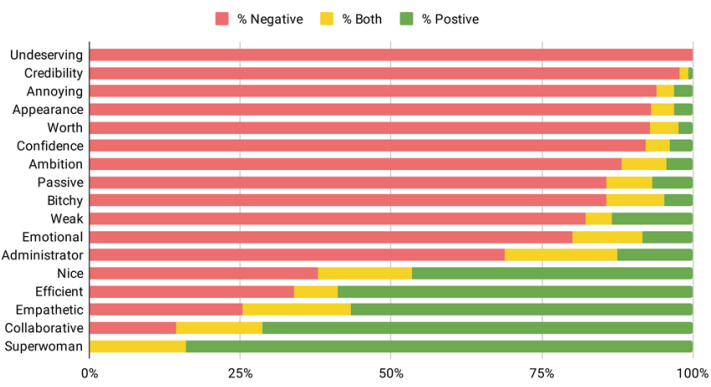

Fig. 1 Perceptions of each stereotype calculated as a percentage of the total responses. Individual focus groups rated each stereotype within the category as either negative, positive or both. Stereotypes are listed from most negatively perceived to least.

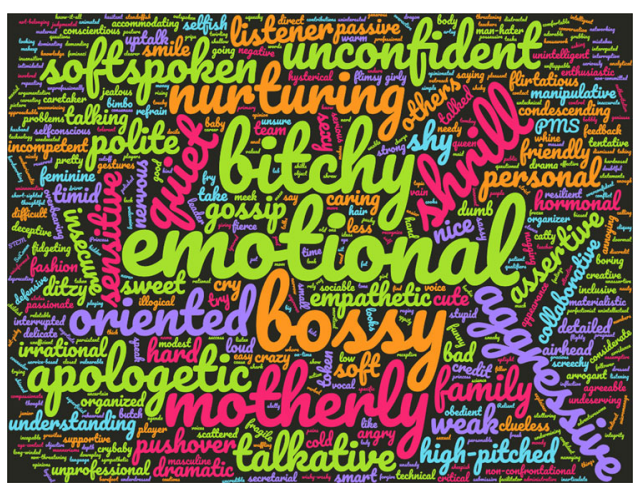

Fig. 2 Word cloud showing most commonly occurring words and phrases elicited via focus groups. The larger the word, the more frequently it appeared in the data. 
they were angry or annoyed at the speaker because they felt the individual perpetuated the stereotype that women are meek and do not have strong opinions. They reacted as if this made it worse for other women, instead of focusing on how they can help the other person be more confident or be heard, or that it might even be a calculated choice. Participants within each workshop were asked for a show of hands whether they had applied the identified negative stereotypes to other women themselves. Consistently 95-100\% of participants at each workshop indicated that they had.

\section{Discussion}

Our results show the top three stereotype categories for women in science who publicly communicate their work as 'Bitchy', lack of 'Credibility', and judged on 'Appearance,' with the specific stereotype words that surfaced the most being 'bossy', 'bitchy', and 'emotional', with 'motherly' in a close fourth. The top perceived stereotypes are consistent with prior studies that found women are judged more on personality and appearance, perceived as less credible than their male colleagues and are expected to be 'warm and nurturing' (Carli et al., 2016; Heilman and Okimoto, 2007; Mitchell and Martin, 2018). Responses to the 'Empathetic' and 'Nice' categories in this study showed that participants had mixed positive and negative associations, which may indicate that women were often judged more harshly when they did not conform to this stereotype, which is consistent with results in earlier studies (Ellemers, 2018; Heilman and Okimoto, 2007; Mitchell and Martin, 2018).

The results also show the majority of stereotypes women face when communicating are perceived to be negative. The negative perception of the stereotypes may be in part due to participants' experience, which is consistent with the literature showing stereotypes perpetuate gender based career disadvantages and behavior expectations for women in STEM (Knobloch-Westerwick et al., 2013; Madera et al., 2009; Weitz, 2018). For example, many burdensome service expectations for female faculty, which are not as valued in promotion and tenure decisions, can often be attributed to the stereotypes that women are more caring, motherly, administrative and outreach oriented. This is supported by the predominantly negative perception of the 'Administrator' category in this study.

Although the categories of 'Nice' and 'Empathetic' were more positively regarded in this study, previous research has shown that being portrayed as 'caring' is not necessarily helpful for career prospects for women in STEM. Women who are seen as career oriented, competent and 'non-traditional' are often deemed competent but not warm, whereas those who are deemed warm are not considered competent (Fiske et al., 2002, 2007) and this might actually negatively affect job prospects when communicated in recommendation letters (Madera et al., 2009). The stereotype categories of lesser credibility, worth and lower confidence all contribute to women being valued less than their male colleagues, as shown in the literature regarding lower salaries, hiring decisions, lower competency ratings, less willingness to mentor, and fewer invitations to speak at conferences (MossRacusin et al., 2012). Negative gender stereotypes perpetuate bias and barriers that persist for women in STEM fields including in career advancement, service burdens, salary, teaching, publishing, speaking roles, promotion and retention. Perceptions of an unfavorable, even unfriendly environment, for women in STEM also has implications for their psychological well-being and subsequent performance (Settles et al., 2016). This then implies that negative stereotypical perceptions do not only negatively affect women, but also the companies and organizations they work for.
Women are often encouraged into role model positions to counteract this institutional and societal bias, and close gender gaps in STEM fields (Diekman et al., 2019). However, this study shows that underlying implicit biases may be actively counteracting any positive contribution to social identity these role models may make. For example, comments elicited during the workshop discussions included women admitting to judging other women more harshly for displaying stereotypical traits such as being soft spoken. This is consistent with earlier research which found members of minoritized groups will punish members of the same group for public displays of potentially negative stereotypes (Opie and Phillips, 2015). Similarly, women showed evidence of stigma consciousness by their acknowledgment of changing their appearance and/or behavior in order to avoid negative judgment and stereotypes.

These results then beg the question: without addressing the underlying gender bias and stereotypical beliefs of individuals, STEM institutions and society, are we putting women at disproportionate risk by encouraging them to be publicly visible role models in STEM? By advocating for more women in STEM as role models, we are asking people who are already minoritized to counteract a whole raft of existing stereotypes, only to have them exposed to many of the same and other stereotypes themselves. Arguably the potential negative impacts are compounded for individuals who may have multiple minoritized group identities. What is experienced by a cisgender, middle class, white female role model in say health or biology, could be vastly different from a transwoman of a culturally diverse background who works in chemistry or engineering for example. Future research should adopt an intersectional lens to gain further understanding of how stereotypes affect STEM professionals in different disciplines and contexts.

In addition, as the lack of fit between female gender-based stereotypes and stereotypes about being a scientist can negatively impact how women in STEM are evaluated, addressing these biases and deeply held stereotypes is necessary to ensure more equitable evaluation (Carli et al., 2016) which has implications for subsequent career progression and potentially the underrepresentation of women at senior levels in STEM fields (Charlesworth and Banaji, 2019). Ellmers (2018) argues that change is unlikely if people do not recognize that stereotypes lie at the root of gender differences in society and work to identify and correct their own bias. Therefore, effective training to address and combat implicit and explicit bias for individuals and institutions is needed, especially for those in positions of privilege and power. Bias training is particularly important given previous studies have shown that when those in positions of power, including but not restricted to men, do not accept evidence of gender bias there is actually a higher level of implicit gender bias in their decision making (Handley et al., 2015; Régner et al., 2019). Previous studies have shown that 'habit-breaking' interventions using strategies such as placing oneself in the perspective of others, thinking of people as individuals rather than part of a group, or exposure to counter stereotypical examples can all be useful to address biases, especially when employed in combination (Charlesworth and Banaji, 2019). It is also important to recognize the utility of this training for women in STEM themselves, with both exposure to female peers and counter-stereotypical role models (male and female) increasing self-efficacy beliefs about success in STEM (Cheryan et al., 2011; Stout et al., 2011)

Although men are more likely to hold positions of power in STEM organizations, a common theme that emerged in the workshops was that it was not just men who were perpetuating the problem. Women felt judged and held back by other women, especially those who had advanced to leadership positions, which supports the findings of an earlier study which showed the 
tendency of minoritized groups to 'police' their own members who exhibit a stereotype (Opie and Phillips, 2015). Simply, women may judge other women more harshly who demonstrate a stereotype, or it may be evidence of the queen bee phenomenon, or more likely it is simply underlying implicit bias. The knowledge of the existence of negative stereotypes, however unfairly labeled, may prevent women from speaking up for fear of being labeled by the stereotype, advocating on behalf of other women or from appearing publicly in a professional capacity at all.

Bias also presented itself in qualifying words like 'too', 'less than' or 'more', which set up an invisible line where a behavior is acceptable, and when it is not. Qualifiers are arbitrary and often differ from person to person. Words like these may encourage an us-versus-them mentality and set up a divide between groups, propagating an environment where the impacts of bias persist and where women are held to a double standard (Rivera and Tilcsik, 2019). Who gets to decide what is 'too much' or 'too little', or whether someone is 'more' or 'less than' someone else? The results of this study imply that there is a preferred amount or level of these characteristics; a tightrope upon which women in particular must balance. However, considering there is not an accepted designation of the 'preferred' amount, it is often dictated by those in a position of power or privilege, shaped by their own bias and stereotypes.

Being aware of the stereotypes presented here, and the implicit and explicit bias they perpetuate, is the first step in combating them. Most people have some level of gendered stereotype bias. A recent study by UNDP showed that worldwide, $91 \%$ of men and $86 \%$ of women hold at least one bias against women with regard to politics, economics, education, violence or reproductive rights (UNDP, 2020). By acknowledging and understanding individual reactions to stereotypes applied to women in STEM, we better understand where our own bias may emerge, and are more equipped to tackle it. It also allows us to more thoroughly examine the impact that being a role model and communicating science publicly has on women in STEM. In addition, understanding stereotypes and our own reactions to them, may enable the development of better mechanisms to support women in STEM who assume role model positions.

This study used conventional qualitative analysis techniques which provide a baseline overview of perceptions of stereotypes, rather than a deeper analysis or development of theory. Future work may wish to build upon the results presented here to develop more fulsome understandings of the relationship between these stereotypes and lived experience of women and other minoritized groups in STEM, which could be further supported through the inclusion of participant demographic data. This would also allow for latent analysis of content to provide deeper and richer data and understanding. The results presented here were collected from people who had chosen to attend, and participate in, a workshop specifically about stereotypes and women in STEM. Future studies may wish to broaden this participant pool to gather perceptions of those not inherently interested in the topic. In addition, as all but one of the workshops was marketed to scientists identifying as women, further studies may wish to include male and non-binary scientists.

\section{Conclusion}

Role models may be held up as a means of addressing prevailing stereotypes, however it appears that the equally important element of inclusion is being ignored. By encouraging women to be visible and communicate their work publicly, and then holding them to double-standards in communication and labeling them as bitchy, bossy, or undeserving, we are failing. By pushing solutions that include more women in STEM as role models, without addressing underlying negative stereotypes they will face, we may actually be putting those women in a more precarious position. Acknowledging and understanding the multifaceted, and often at odds, stereotypes that women in STEM face when communicating in public is the first step in changing the narrative and developing better mechanisms to support women in STEM.

The results presented in this study show that the prevailing perceptions of the stereotypes applied to women who speak publicly about their work are largely negative. These are the perceptions of women who, through participating in this study, are showing an interest in matters of equity and diversity. Any attempts to support greater gender equity and inclusion then must also acknowledge our own biases and deeply held stereotypes and recognize how they might affect our behavior and judgment regarding ourselves and others. Creating networks of support for women and giving them tools and space to address gender bias in STEM can help in battling discrimination. However, to truly change the system we must train everyone, including ourselves and especially those in positions of power and privilege, to be effective bystanders against gender bias, sexual harassment, and the perpetuation of negative stereotypes.

\section{Data availability}

The datasets generated during and/or analyzed during this study are not publicly available due to the potential for individuals/ groups to be identified, but are available from the corresponding author on reasonable request.

Received: 20 April 2020; Accepted: 3 November 2020; Published online: 25 November 2020

\section{References}

Amarasekara I, Grant WJ (2019) Exploring the YouTube science communication gender gap: a sentiment analysis. Public Underst Sci 28:68-84

Bendels MHK, Müller R, Brueggmann D, Groneberg DA (2018) Gender disparities in high-quality research revealed by Nature Index journals. PLoS ONE 13: e0189136

Bian L, Leslie SJ, Cimpian A (2017) Gender stereotypes about intellectual ability emerge early and influence children's interests. Science 355:389-391

Budden AE, Tregenza T, Aarssen LW, Koricheva J, Leimu R, Lortie CJ (2008) Double-blind review favours increased representation of female authors. Trends Ecol Evol 23:4-6

Cadaret MC, Hartung PJ, Subich LM, Weigold IK (2017) Stereotype threat as a barrier to women entering engineering careers. J Vocat Behav 99:41-50

Carli LL, Alawa L, Lee YA, Zhao B, Kim E (2016) Stereotypes about gender and science: women \# scientists. Psychol Women Quart 40(2):244-260

Carlone HB, Johnson A (2007) Understanding the science experiences of successful women of colour: science identity as an analytic lens. J Res Sci Teach 44:1187-1218

Charlesworth TES, Banaji MR (2019) Gender in science, technology, engineering and mathematics: issues, causes, solutions. J Neurosci 39:7228-7243

Cheryan S, Siy JO, Vichayapai M, Drury BJ, Kim S (2011) Do female and male role models who embody STEM stereotypes hinder women's anticipated success in STEM? Soc Psychol Pers Sci 2:656-664

Chimba M, Kitzinger J (2010) Bimbo or boffin? Women in science: an analysis of media representations and how female scientists negotiate cultural contradictions. Public Understand Sci 19:609-624

Cho JY, Lee E (2014) Reducing confusion about grounded theory and qualitative content analysis: similarities and differences. Qual Rep 16:Art. 64

Cimpian JR, Lubienski ST, Timmer JD, Makowski MB, Miller EK (2016) Have gender gaps in math closed? Achievement, teacher perceptions, and learning behaviors across two ECLS-K cohorts. AERA Open 2:2332858416673617

Clancy KBH, Lee KMN, Rodgers EM, Richey C (2017) Double jeopardy in astronomy and planetary science: Women of color face greater risks of gendered and racial harassment. J Geophy Res 122:1610-1623

Derks B, Van Laar C, Ellemers N (2016) The queen bee phenomenon: why women leaders distance themselves from junior women. Leadership Quart 27:456-469

Diekman AB, Clark EK, Belanger AL (2019) Finding common ground: synthesizing divergent theoretical views to promote women's STEM pursuits. Soc Issues Policy Rev 13:182-210 
Drury BJ, Siy JO, Cheryan S (2011) When do female role models benefit women? The importance of differentiating recruitment from retention in STEM. Psychol Inquiry 22:165-269

Ellemers N (2018) Gender stereotypes. Ann Rev Psychol 69:275-298

Elo S, Kyngäs H (2008) The qualitative content analysis process. J Adv Nursing 62:107-115

Faulkner W (2009) Doing gender in engineering workplace cultures: gender in/ authenticity and the in/visibility paradox. Eng Stud 1:169-189

Fiske ST, Cuddy AJC, Glick P (2007) Universal dimensions of social perception: warmth and competence. Trends Cogn Sci 11:77-83

Fiske ST, Cuddy AJC, Glick P, Xu J (2002) A model of (often mixed) stereotype content: competence and warmth respectively follow from perceived status and competition. J Personal Soc Psychol 82:878-902

Garriott PO, Hultgren KM, Frazier J (2017) STEM stereotypes and high school students' math/science career goals. J Career Assess 25:585-600

Graneheim UH, Lundman B (2004) Qualitative content analysis in nursing research: concepts, procedures and measures to achieve trustworthiness. Nurse Educ Today 24:105-112

Handley IM, Brown ER, Moss-Racusin CA, Smith JL (2015) Quality of evidence revealing subtle gender biases in science is in the eye of the beholder. Proc Natl Acad Sci USA 112:13201-13206

Heilman ME, Okimoto TG (2007) Why are women penalized for success at male tasks?: the implied communality deficit. J Appl Psychol 92:81-92

Hsieh H, Shannon SE (2005) Three approaches to qualitative content analysis. Qual Health Res 15:1277-1288

Knobloch-Westerwick S, Glynn CJ, Huge M (2013) The Matilda Effect in science communication: An experiment on gender bias in publication quality perceptions and collaboration interest. Sci Commun 35:603-625

Konik J, Cortina LM (2008) Policing gender at work: Intersections of harassment based on sex and sexuality. Soc Justice Res 21:313-337

Lindkvist K (1981) Approaches to textual analysis. In: Rosengren KE (ed) Advances in content analysis. SAGE, Beverly Hills

Madera JM, Hebl MR, Martin RC (2009) Gender and letters of recommendation for academia: agentic and communal differences. J Appl Psychol 94:1591-1599

Manning K (1997) Authenticity in constructivist inquiry: Methodological considerations without prescription. Qual Inquiry 3:93-115

Mason J (2002) Qualitative researching. Sage, London

Mayring P (2000) Qualitative content analysis. Qual Soc Res 1:Art. 20, http://nbnresolving.de/urn:nbn:de:0114-fqs0002204

Miles MB, Huberman AM (1994) Qualitative data analysis: an expanded sourcebook, 2nd edn. Sage, Newbury Park

Milkman KL, Akinola M, Chugh D (2015) What happens before? A field experiment exploring how pay and representation differentially shape bias on the pathway into organizations. J Appl Psychol 100:1678-1712

Mitchell KMW, Martin J (2018) Gender bias in student evaluations. Politi Sci Polit 51:648-652

Moss-Racusin CA, Dovidio JF, Brescoll VL, Graham MJ, Handelsman J (2012) Science faculty's subtle gender biases favor male students. Proc Natl Acad Sci USA 109:16474-16479

National Academies of Sciences Engineering and Medicine (2018) Sexual harassment of women: climate, culture, and consequences in academic sciences, engineering, and medicine. National Academies of Sciences Engineering and Medicine, Washington, DC

Niemi MK, Pitkänen V (2017) Gendered use of experts in the media: analysis of the gender gap in Finnish news journalism. Public Understand Sci 26:355-368

Noy S, O'Brien TL (2019) Science for good? The effects of education and national context on perceptions of science. Public Understand Sci 28:897-916

O’Dea RE, Lagisz M, Jennions MD, Nakagawa S (2018) Gender differences in individual variation in academic grades fail to fit expected patterns for STEM. Nat Commun 9:3777

Opie TR, Phillips KW (2015) Hair penalties: the negative influence of Afrocentric hair on ratings of Black womenas dominance and professionalism. Front Psychol 6:1311

Pinel EC (1999) Stigma consciousness: the psychological legacy of social stereotypes. J Personal Soc Psychol 76:114-128
Régner I, Thinus-Blanc C, Netter A, Schmader T, Huguet P (2019) Committees with implicit biases promote fewer women when they do not believe gender bias exists. Nat Human Behav 3:1171-1179

Rivera LA, Tilcsik A (2019) Scaling down inequality: rating scales, gender bias, and the architecture of evaluation. Am Sociol Rev 84:248-274

Roberts LM, Settles IH, William AJ (2008) Predicting the strategic identity management of gender and race. Identity 8:269-306

Rosenthal MN, Smidt AM, Freyd JJ (2016) "Still second class: sexual harassment of graduate students": corrigendum. Psychol Women Quart 40:NP1

Sandelowski M (2000) Focus on research methods: whatever happened to qualitative description? Res Nurs Health 23:334-340

Settles IS, O'Connor RC, Yap SCY (2016) Climate perceptions and identity interference among undergraduate women in STEM: The protective role of gender identity. Psychol Women Quart 40:488-503

Shin JEL, Levy SR, London B (2016) Effects of role model exposure on STEM and non-STEM student engagement. J Appl Soc Pyschol 46:410-427

Smyth FL, Nosek BA (2015) On the gender-science stereotypes held by scientists: explicit accord with gender-ratios, implicit accord with scientific identity. Front Psychol 6:415

Stout JG, Dasgupta N, Hunsinger M, McManus MA (2011) STEMing the tide: using ingroup experts to inoculate women's self-concept in science, technology, engineering, and mathematics (STEM). J Pers Soc Psychol 100:255-270

Steele CM (1997) A threat in the air: How stereotypes shape intellectual identity and performance. Am Psychol 52:613-629

UNDP (2020) 2020 human development perspectives: tackling social norms. A game changer for gender inequalities. United Nations Development Programme, New York

Veletsianos G (2012) HIgher education scholars' participation and practices on Twitter. J Comput Assisted Learn 28:336-349

Weitz A (2018) Sexual harassment is rife in the sciences, finds landmark US study: existing policies to address the issue are ineffective, concludes a long-awaited report from the National Academies of Sciences, Engineering, and Medicine. Nature 558:352-353

Women's Leadership Institute Australia (2019) Women for Media Report. https:// docs.wixstatic.com/ugd/ee1ce5_88c20ce959044aab84737b1993c326ca.pdf

\section{Competing interests}

The authors declare no competing interests.

\section{Additional information}

Correspondence and requests for materials should be addressed to M.M.

Reprints and permission information is available at http://www.nature.com/reprints

Publisher's note Springer Nature remains neutral with regard to jurisdictional claims in published maps and institutional affiliations.

\begin{abstract}
Open Access This article is licensed under a Creative Commons Attribution 4.0 International License, which permits use, sharing, adaptation, distribution and reproduction in any medium or format, as long as you give appropriate credit to the original author(s) and the source, provide a link to the Creative Commons license, and indicate if changes were made. The images or other third party material in this article are included in the article's Creative Commons license, unless indicated otherwise in a credit line to the material. If material is not included in the article's Creative Commons license and your intended use is not permitted by statutory regulation or exceeds the permitted use, you will need to obtain permission directly from the copyright holder. To view a copy of this license, visit http://creativecommons.org/ licenses/by/4.0/.
\end{abstract}

(C) The Author(s) 2020 